\title{
A Historical Approach to Teaching Work and Energy in Upper Secondary Education
}

\section{Jarier Wannous ${ }^{\text {a }}$, Peter Horváth ${ }^{\text {b }}$}

Comenius University in Bratislava, Bratislava, Slovakia, jarier.wannous@,fmph.uniba.sk, beter.horvath@fmph.uniba.sk

\begin{abstract}
In the context of three-dimensional learning, it is important for students to discover new concepts through different activities and experiments. The most common approach to teaching work and energy is defining work using intuitive ideas of students and then continuing by deriving potential and kinetic energy from work. In our paper, we have decided to analyze how the concepts of work and energy, as understood in mechanics, developed in history, while concentrating on experiments that lead to their discovery. Using facts learned from our historical analysis, we propose a different approach to introducing the concepts of work and energy based on discovery through experiments. The activities used in our approach are discussed in detail in our paper, where they are arranged to parallel the historical development of the aforementioned concepts. We believe the approach can offer teachers an alternative where students can discover work and energy on their own and understand their value in physics.
\end{abstract}

Keywords: Work, Energy, Historical approach, Three-dimensional learning, mechanics.

\section{Introduction}

One of the classical ways of teaching work and energy found in physics textbooks today is starting with defining work done by a constant force and then continuing by showing the relationship between work, kinetic and potential energy. Take for example Giancoli's Physics: Principles with Applications (2014). In the sixth chapter he discusses work and energy and then divides the chapter into the following subchapters:

- Work Done by a Constant Force.

- Work done by a Varying Force.

- $\quad$ Kinetic Energy, and the Work-Energy Principle. 
- Potential Energy.

- Conservative and Nonconservative Forces.

- Mechanical Energy and Its Conservation.

Our work in the past (Wannous, 2017) has led us to use a historical approach in our teaching of certain concepts in mechanics with a fair amount of success. This means that when teaching those concepts, we studied the history of their development, we analyzed key moments in that history and we used them to propose a sequence of activities focused on teaching those concepts similarly to the way they developed in history. In this work we propose using this approach with the concepts of work and energy as they are understood in mechanics.

That is why we first discuss the concepts of work and energy in the context of threedimensional learning, we analyze the history of the aforementioned concepts and highlight the differences between the historical development and the classical method of their teaching, then based on those differences we propose a sequence of activities that can be used to teach the concepts of work and energy.

\section{Three-Dimensional Learning and Energy}

Before closely discussing the teaching plan of work and energy, we take a quick look at the report Framework for K-12 Science Education: Practices, Crosscutting Concepts, and Core Ideas (the Framework) prepared by the National Research Council (2012). In the proposed frame work the national council recommends building science education around three major dimensions:

1. Scientific and engineering practices

2. Crosscutting concepts

3. Disciplinary core ideas

The first dimension of the framework describes the main practices that scientists and engineers employ while investigating, designing, building models or systems. Bybee (2011) listed the practices students are supposed to know and be able to do as following:

- Asking questions and defining problems.

- Developing and using models.

- Planning and carrying out investigations.

- Analyzing and interpreting data.

- Using mathematics and computational thinking.

- Constructing explanations and designing solutions. 
- Engaging in argument from evidence.

- Obtaining, evaluating, and communicating information.

Most of these practices can be developed in the classroom simply by using a fair amount of experiments and activities. Of course, when talking about a plan for teaching work and energy, we must keep in mind that it is only a part of a larger subject. This means that although using activities while teaching work and energy can help develop scientific and engineering practices, it does not mean that we expect them to be enough on their own, but that they are a part of a larger plan of teaching mechanics.

The second dimension of the framework deals with concepts that can be found across different disciplines in science, and so they bridge those disciplines. Duschl (2012) gives a comprehensive list of the crosscutting concepts given in the framework as following:

- Patterns.

- Cause and Effect: Mechanism and Explanation.

- $\quad$ Scale, Proportion, and Quantity.

- Systems and System Models.

- $\quad$ Energy and Matter: Flows, Cycles, and Conservation.

- Structure and Function.

- $\quad$ Stability and Change.

It is noticeable that the subject of work and energy contains in it most, if not all, of the mentioned crosscutting concepts. We will see in the analysis of the historical development of work and energy how these crosscutting concepts fit into the narrative. This helps us later in recognizing the proper place of each crosscutting concept in the teaching plan.

The last dimension of the framework deals with core ideas specific to each discipline. As the list of disciplinary core ideas given by the framework (National Research Council, 2012) is extensive, we list only those core ideas which can be developed throughout our plan. The list of the core ideas divided by discipline is as following:

\section{- Physical Sciences:}

- Energy: the transfer of energy from one object to another is an important mechanism needed to explain and predict interactions between objects. That is why this core idea is divided in to the following component concepts:

- Definition of energy: energy as a quantitative property of a system depending on motion and interactions. Energy can manifest itself in multiple ways, such as in motion, sound, light, and thermal energy. 
- Conservation of energy and energy transfer: A system's total energy is conserved. This conservation can be used to predict and describe system behavior.

- Relationship between energy and forces: Force fields contain energy and can transmit energy across space from one object to another.

- Life Sciences:

- Life sciences contain two core ideas which can be connected to our subject: From Molecules to Organisms: Structures and Processes and Ecosystems: Interactions, Energy, and Dynamics. Some of the component concepts of these core ideas are directly connected with the conservation of energy. One of the characterizations, for example, states that "matter and energy are conserved in each change. This is true of all biological systems, from individual cells to ecosystems" (National Research Council, 2012). From the point of view of physics, this is not only true for biological systems but also for any closed system.

- $\quad$ Earth and Space Sciences:

- This discipline does not contain any core ideas or component concepts which can be directly linked with our plan.

- Engineering Design:

- The core ideas of this discipline are connected closely to the practices mentioned earlier in this article. However, it also deals with the connection between science and engineering. This connection can also be a part of our subject, especially when we deal with power and how efficiency is an important factor in engineering design.

As we can see from our quick analysis of the framework, three-dimensional learning can be, and should be, integrated into our teaching plan of work and energy. Our plan will be focused on the correct development of core ideas connected with work and energy, as well as the development of crosscutting concepts and scientific and engineering practices.

\section{The History of Work and Energy}

As we have said earlier in our paper, our plan will be based on the historical development of the concepts of work and energy. In this historical analysis, we will not delve into the history of heat and its connection with mechanical energy, but will focus only on mechanical energy, i.e. the concepts of kinetic and potential energy as well as work and power. 
The history of mechanical energy starts with the rivalry between Leibnitz and Newton. Leibnitz did not agree with Newton's definition of Force and with his three laws of motion, so he worked to develop a different theory to explain forces. Instead of defining force like Newton using the change of momentum as following:

$$
F=\frac{d p}{d t}
$$

He proposed that forces can be divided into two types (Iltis, 1971):

- Vis mortua or dead force. There was some confusion about the meaning of dead force in the time of Leibnitz. Mainly this quantity was sometimes understood as momentum $(\mathrm{mv})$, and in other times it was understood as $(\mathrm{mdv})$. The latter comes close to the meaning of force as given by Newton. In general, a dead force is an elementary force, which do not contain motion. Or in Leibnitz's own words: "The elementary force, which I call dead because motion does not yet exist in it, but only a solicitation to motion, is like that of a sphere in a rotating tube or a stone in a sling... . Examples of dead force are provided by centrifugal force, by gravity or centripetal force, and by the force with which a stretched spring starts to contract" (Dugas, 1955).

- Vis Viva or living force. This force is directly associated with motion. Leibnitz understood it to be an infinity of impressions of dead force: "But in percussion that is produced by a body which has been falling for some time, or by an arc which has been unbending for some time, or by any other means, the force is living and born of an infinity of continued impressions of the dead force" (Dugas, 1955).

Leibnitz defined living force as the quantity:

$$
F_{l}=m v^{2}
$$

Which, if we ignore the constant $\frac{1}{2}$, is identical to our modern concept of kinetic energy. An interesting fact is that if we use Leibnitz's understanding of the connection between living and dead forces, we will be able to calculate dead force as following:

$$
\begin{gathered}
F_{l}=\int F_{d} \cdot d s \\
d\left(m v^{2}\right)=F_{d} \cdot d s \\
F_{d}=2 m \frac{d v}{d t}
\end{gathered}
$$

This might lead us to the conclusion that Leibnitz's understanding of dead forces is similar, if not identical (if we ignore the constant just like in kinetic energy), to Newton, even though Leibnitz would probably deny such a fact. 
Leibnitz was convinced on metaphysical grounds that living force is conserved in nature. His main example for showing this conservation were elastic collisions (Boudri, 2002). This conservation, he argued, is superior to the conservation of motion or the value $m v$. One of his arguments for this assertion was for example (fig. 1) that "the same force is necessary to raise a body A of 1 pound [libra] to a height 4 yards [ulnae] as is necessary to raise a body B of 4 pounds to a height of 1 yard" (Iltis, 1971). Then he argued that according to Galileo body A will acquire in its fall twice the velocity as body B, which means that the values of momentum will not be the same, whereas living force will be.

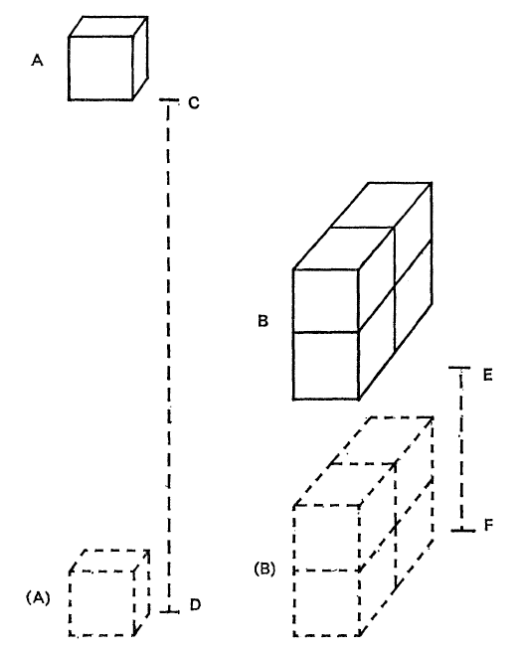

Figure 1. Leibnitz's example. Source: (Iltis, 1971)

Leibnitz's argument for conservation of living force is important, for it shows us that not only Leibnitz was extremely close to the concept of kinetic energy, but also to the concept of potential energy in a uniform gravitational field, if not the concept of work. If nothing else, we can see that Leibnitz acknowledged that living force is transferred into acquiring height in vertical throws.

The next milestone in this subject comes with the work of Willem Gravesande (Boudri, 2002), who experimented with copper balls falling into clay in the 1720s. He showed that the depth of the hollows made by the balls was proportional to the quantity $m v^{2}$. This experiment proved the conservation of living force, but we believe its implications can be expanded into connecting energy and force (work) as well.

The term living force was used for years later, and although the term "energy" was first used by Thomas Young in 1807, and the term "kinetic energy" was described by GustaveGaspard Coriolis in 1829, the term was not accepted until it was popularized by William Thompson (Lord Kelvin) and William J. M. Rankine in 1851 - 1852. 
The history of the concept of energy reveals some important points which we can use throughout our plan:

- A classical approach to teaching mechanics starts by defining work using force, then continues to derive kinetic and potential energy out of work. The history of energy does not follow a similar progression.

- In history, energy developed as an opposing theory to force as it was defined by Newton. The connection between the two was recognized only later.

- The concept of kinetic energy was observed first based on the observations of elastic collisions. Later the concept of potential energy in a uniform gravitation field was developed based on vertical throws.

- Gravesande's experiment with balls and clay can create a connection between force and kinetic (or potential) energy, on the basis of proportionality between energy and depth of the hollows made by balls.

- The whole development of the concepts of work and energy was based on some of the crosscutting concepts as mentioned by the framework. Kinetic energy was seen through patterns in elastic collisions, while potential energy was the effect of kinetic energy (cause) in vertical throws.

We will use these points in the following part of our article to create a teaching plan based on the historical development of work and energy.

\section{Teaching Work and Energy}

As we have said earlier, our teaching plan will be based on the historical development of the taught concepts, while we still keep in mind the requirements we need to fulfill from the framework. We will describe the key activities needed for the plan.

Just as in history, we propose to introduce energy starting with kinetic energy based on the principle of conservation in elastic collisions. If teaching mechanics starts with the subject of force and motion, either in a classical way of teaching or in the way we proposed in our earlier work (Horváth \& Wannous, 2017), then it means students should have a grasp on the concept of conservation based on the conservation of momentum. Therefore, the first activity in our teaching plan will be defining kinetic energy using elastic collisions. For an introduction of video analysis, we recommend Experiments and Video Analysis in Classical Mechanics (Jesus, 2014).

Activity 1. Video analysis of elastic collisions: Using video analysis of elastic collisions of carts, students can discover the value $m v^{2}$, which later can be defined as kinetic energy. Although it is important to note that this activity requires some guidance from the teacher. The teacher does not have to tell the students that they should try $m v^{2}$ right away, but he 
can use the fact that students already know about the conservation of momentum, and he can reveal that they should try the powers of those quantities.

This activity has one problem though, and that is that the conservation of energy requires perfectly elastic collisions, which is hard to achieve, especially when those collision are not in a closed system. To deal with this problem, we recommend teachers prepare the videos of the collisions instead of students, and then give them the final product.

After discovering the conservation of the quantity $m v^{2}$, we can define it as kinetic energy (after a small change by adding the constant $\frac{1}{2}$, which the teacher can explain later after defining work). Then, we can proceed to discover potential energy in a uniform gravitational field, which can be done by another video analysis.

Activity 2. Video analysis of vertical throw: In this activity students are supposed to form a hypothesis about what will happen to the kinetic energy at the beginning of the throw and then prove that hypothesis. This activity can first lead to the identification that maximum height is proportional to kinetic energy, which will lead to the identification and definition of potential energy in a uniform gravitational field. A full analysis of vertical throw should lead to the fact that potential energy is interchangeable with kinetic energy, which can lead to the definition of the law of conservation of energy.

After defining kinetic and potential energy, the final step is to connect energy to force by defining work. For this, we propose using Gravesande's experiment with balls falling into clay.

Activity 3. Balls falling into clay: Rather than connecting the depth of the hollow made by the ball, we propose that student investigate the relationship between potential energy and depth. However, the experiment can be given an open question such as finding the relationship between the energy and the depth of the hollow.

Finding the relationship between potential energy and depth should lead students to a proportionality between the two quantities, which can offer further grounds for the investigation of the phenomena, such as other quantities that can influence the depth of the hollow. This should the students to defining work as new quantity.

After defining work and energy, the only thing left is dealing with power. This brings us to two final activities.

Activity 4. Hiring workers: The students get into the role of a construction manager, who is supposed to hire a new worker when given two options. The student has to decide which one he would hire in three different cases: 
a) The workers do different amounts of works at the same time.

b) The workers do the same amount of work in different times.

c) The workers do different amounts of work in different times.

This simple activity should lead the students to discover power as a physical quantity on their own.

Activity 5. Measuring students' individual power: students measure their own power while going up the stair to the highest floor in the school. Other than experiencing power on their own, this activity has a pleasant side effect. Each student has their own individual mass, some may be higher and others lower, however their performance while going up the stairs to the highest floor will be similar. This will lead to students having the highest mass to have the highest power. This can be helpful, especially in today's higher occurrence of fat shaming in high schools, although an indirect one that need not be stressed.

A quick look at the activities mentioned in our plan can show, that not only they are based on history, but with correct implementation in the classroom, they can conform with threedimensional learning as characterized by the framework.

\section{Conclusion}

Based on our earlier experience with historical approach, we have decided to develop a plan for teaching the concepts of work and energy. The developed plan was created according to the criteria given by the framework. We started by analyzing the history of the concepts of work and energy, where we found a few interesting points which do not fit with a classical teaching sequence. One of them for example was that kinetic energy was the first discovered of the concepts and work came until much later. After the historical analysis we offered a plan proposal. There we can find experiments which parallel the points we found in history, and still help develop the dimensions expressed in the framework.

This plan will be tested throughout 2018-2020 as a part of a general plan for teaching mechanics. In our future testing, we will not only concentrate on concept understanding, but we will try to see how other aspects of learning has been affected by our plan, such as scientific and engineering practices for example. We believe that this plan will make a great addition to our original mechanics plan but can be also helpful for any teacher who is looking for an effective way to teach work and energy. 


\section{References}

Boudri, J. C. (2002). What was Mechanical about Mechanics: The Concept of Force between Metaphysics and Mechanics from Newton to Lagrange. Springer Science+Business Media Dordrecht.

Bybee, R. W. (2011). Scientific and Engineering Practices in K-12 Classrooms: Understanding A Framework for K-12 Science Education. Science Teacher, 34-40.

Dugas, R. (1955). A History of Mechanics. London.

Duschl, R. A. (2012). The Second Dimension--Crosscutting Concepts. Science Teacher, 34-38.

Giancoli, D. C. (2014). Physics: Principles with Applications. Pearson Education.

Hestenes, D., \& Halloun, I. (1995). Interpreting the Force Concept Inventory. The Physics Teacher, 502-506.

Horváth, P., \& Wannous, J. (2017). Hisotircký prístup vo vyučovaní pojmu sila na gymnáziu. Tvorivý učitel fyziky $X$. Smolenice.

Iltis, C. (1971). Leibnitz and the Vis Viva Controversy. Isis, 21-35.

Jesus, V. L. (2014). Experiments and Video Analysis in Classical Mechanics: Undergraduate Lecture Notes in Physics. S ao Paulo.

National Research Council. (2012). A Framework for K-12 Science Education: Practices, Croscutting Concepts, and Core Ideas. Washington, DC: The National Academies Press.

Wannous, J. (2017). Od pojmu hybnost' k pojmu sila vo vyučovani fyziky na gymnáziu. Bratislava. 